\title{
A Parallel Energy-Sharing Control for Fuel cell- Battery-Ultracapacitor Hybrid Vehicle
}

\author{
JennHwa Wong, N.R.N.Idris , Makbul Anwari, Taufik Taufik
}

\begin{abstract}
This paper proposes a parallel energy-sharing control for fuel cell hybrid vehicles (FCHVs) application. The hybrid source consists of fuel cells (FCs) stack, battery packs and Ultracapacitor (UC) modules. In the proposed parallel energy sharing control, each source is connected to a DC bus via power electronics converters. A total of six control loops are applied in the supervisory system in order to regulate the DC bus voltage, control of power flow and at the same time to monitor the state of charge (SOC) of each energy storage device. Simulation with experiment verifications are carried out to verify the proposed energy control system.
\end{abstract}

\section{INTRODUCTION}

Due to the rapid escalating of gasoline prices, depletion of fossil fuels and environmental concerns, fuel cell hybrid vehicles (FCHVs) are now gaining popularity among the automotive companies around the world. Application of FCs as the primary energy source in a vehicle is seen as one of the promising solutions to provide a high energy efficient, quiet, less pollutant and .longer travel range (as long as the fuel supply is available). However, there are several problems faced when one tries to use FC alone to power the vehicle, these include poor dynamic response, difficulty during FC cold startup, high cost, and inability to capture braking energy during vehicle decelerations or downhill [1]-[3]. Moreover, peak power demand from $\mathrm{FC}$, for example during vehicle acceleration, could lead to fuel starvation phenomenon and shorten its lifespan [1],[2]. For these reasons, hybridization of FC with energy storage units (ESUs) is necessary in order to overcome these problems as well to reduce the vehicle size and cost [2].

ESUs could comprise of battery modules alone, UC modules or the combination of both battery and UC modules (combined-ESUs). A comparative study performed in $[2,4,5]$ indicated that FC-battery-UC hybrid vehicle could lead to a more practical solution, higher fuel economy and longer battery lifespan. From the literatures, most of the proposed energy management strategies applied in the FC hybrid source are of series energy flow configurations[1,2,6] where the energy dense source is used to charge the power dense source, and the power dense source is used to regulate the DC voltage bus as well as to response to peak power demand.

This paper proposed a parallel energy-sharing control strategy for FCHV with FC generator as the primary energy source and a combination of battery and UC as the ESUs. In the parallel energy-sharing strategy, the power flow between ESUs and FC is controlled in order to stabilize the DC bus voltage. Unlike the series energy flow configuration, the DC bus is simultaneously regulated by FC and ESUs however, with different share of contribution depending on the characteristics of sources and the mode of operations. In other words, the parallel energy control can provide higher degree of energy sharing, increased flexibility and greater output power during steady state and transient. Consequently, the overall volume of the hybrid sources can be downsized and optimized. The battery, on the other hand, can be operated in a narrow chargedischarge cycle and can be in idle state during steady state vehicle operation provided that the based power demand can be fulfilled by the FC generator. The aim of the paper is to discuss on the control structures and the design of the proposed parallel energy-sharing algorithm. However, it is not the scope of this paper to discuss on the selection and sizing of these energy sources.

Arrangement of the paper is as follows: section 2 discusses on the energy management design while section 3 discusses on the proposed control strategy. The results are outlined in section 4 together with discussions. Lastly, the conclusion is presented in Section 5.

\section{ENERGY MANAGEMENT DESIGN}

Energy management is one of the most important factors to ensure the optimization in efficiency, excellence dynamic performance and reliability of a FCHV. This is true especially in a combined-ESUs system. In order to optimally use the energy sources and ensure safety operation, the proposed algorithm in this paper is developed based on the 
characteristics of vehicle load components, FC, UC and battery. These are discussed as follows.

- FCHV load components can be categorized into two types: constant load and transient load. Constant load consists of based load (on-board electric load and air conditioning), rolling resistance, aerodynamic drag and gravitational load during uphill or downhill. These loads are almost constant and basically does not contain any peak power demand. These types of loads are compatible with the inherent characteristics of the FC generator, which has a slow response to power demand. On the other hand, transient load is associated with the power needed during acceleration, deceleration or braking that causes peak power transient. FC alone will not be able to respond to this type of power demand and thus ESUs will be used to compensate this.

- Fuel cell (FC) shows slow transient response and has relatively high internal resistance. FC system has the disadvantages of slow start-up and this is often cited as one of the major barriers to the use of FC in domestic vehicle especially with the used of fuel reformer [7]. However, FC is able to supply the power continuously for as long as the reactants are available. Hence, it could function as a power generator in the hybrid system by constantly supplying the average or required steady state power to the vehicle; this mode of operation is shown in Fig. 1(a). Since the efficiency of the FC is optimum at high current demand, when the vehicle is running at light load, FC can also be used to charge the battery or the UC depending on the SOC of these ESUs. A power slope limiter is needed to avoid FC from any peak transient response which could cause permanent damage to the cell (FC starvation phenomenon).

- $\quad$ Ultracapacitor (UC) has a very high capacitance density and able to provide a large amount of power (high specific power) within a relatively short period (low specific energy). It is a robust device with extremely long lifecycle, low maintenance and low internal resistance. With these characteristics, UC is suitable for compensating the slow transient response of the $\mathrm{FC}$ during vehicle acceleration, as depicted in Fig 1(b). To limit the large charging current of the battery, the UC is also used to capture the large braking power during deceleration. On the other hand, UC is known to have a relatively low energy density and fast self-discharge characteristics. Application of UC solely as the ESU in FCHV may face start-up problem once the energy content in the UC is depleted due to its self-discharge process.

- Battery has an advantage of high specific energy but relatively low in specific power. The power response is faster than FC, but slower than UC. Furthermore, battery has a limited lifespan (300-2000 cycles) [7-9] depending on the types of the battery, depth of discharge cycles, discharge rate, cell operating temperature, charging regime, number of overcharge and others. Hence, to optimize the lifespan of the battery, it is recommended that the battery current slope must be limited within the safe value in order to reduce peak transient stress. In this case, the peak power demand will be responded by the UC. During the cold start-up, the battery will provide the energy because of the slow response of the $\mathrm{FC}$ and also the fast self-discharge characteristic of the UC, as shown in Fig. 1 (c).

\section{PROPOSED ENERGY CONTROL STRATEGY}

A parallel energy-sharing strategy is proposed to control the power flow between the DC bus (load), primary energy source (FC generator) and ESUs (UC and battery). A parallel active connection between all energy sources and the DC bus as depicted in Fig. 2. In the proposed configuration, the FC is connected to the DC bus via a single quadrant boost converter to step-up it's output voltage and blocks the regenerative power from flowing back into the FC. On the other hand, the battery and UC are connected via the bi-directional power flow halfbridge converters. These three energy sources will be used simultaneously to regulate the DC bus voltage.

As shown in Fig. 3, the control structure of the proposed parallel energy-sharing control contained a total of six control loops (all are using PI-controllers). These included of a DC bus voltage control loop, three inner current control loops, an UC voltage control loop and a battery charging control loop.

a)

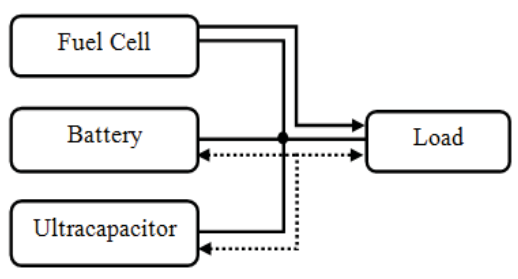

b)

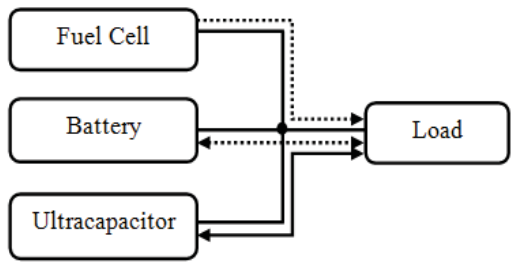

c)



Fig. 1 Mode of power flow in the proposed hybrid system: a) during steady state, b) during transient, c) during start-up (solid arrow indicates of main power flow, dotted arrow indicates of secondary power flow)

The DC bus voltage control loop is used to control the DC bus output voltage to its fixed reference value. A current 
reference $\left(I_{\text {load }}\right)$ signal is generated by the DC bus voltage controller and becomes the main source of the three inner current control loop references namely the FC, the battery and the UC current loops. The switching frequency of the PWM current loops is set to $10 \mathrm{kHz}$, hence with this, the cut-off frequencies for the voltage and current control loops are selected as $10 \mathrm{~Hz}$ and $1 \mathrm{k} \mathrm{Hz}$ respectively. In order to limit the slope of the current references for the battery and FC within their safe values, low pass filters with time constants $\tau_{1}$ and $\tau_{2}$ $\left(\tau_{2}<\tau_{1}\right)$ are used respectively. The final value of current reference for the $\mathrm{UC}$ is obtained by subtracting the reference current generated by the DC voltage loop with the output current from battery and FC; this is to ensure that only the UC current reference contains the demanding peak transient elements of the load current reference. To enable the battery to operate in a narrow charge-discharge cycle, the battery current reference is subtracted with the FC output current. By doing so, the peak power demand from the FC is avoided and at the same time only the FC will supply the continuous steady state power.

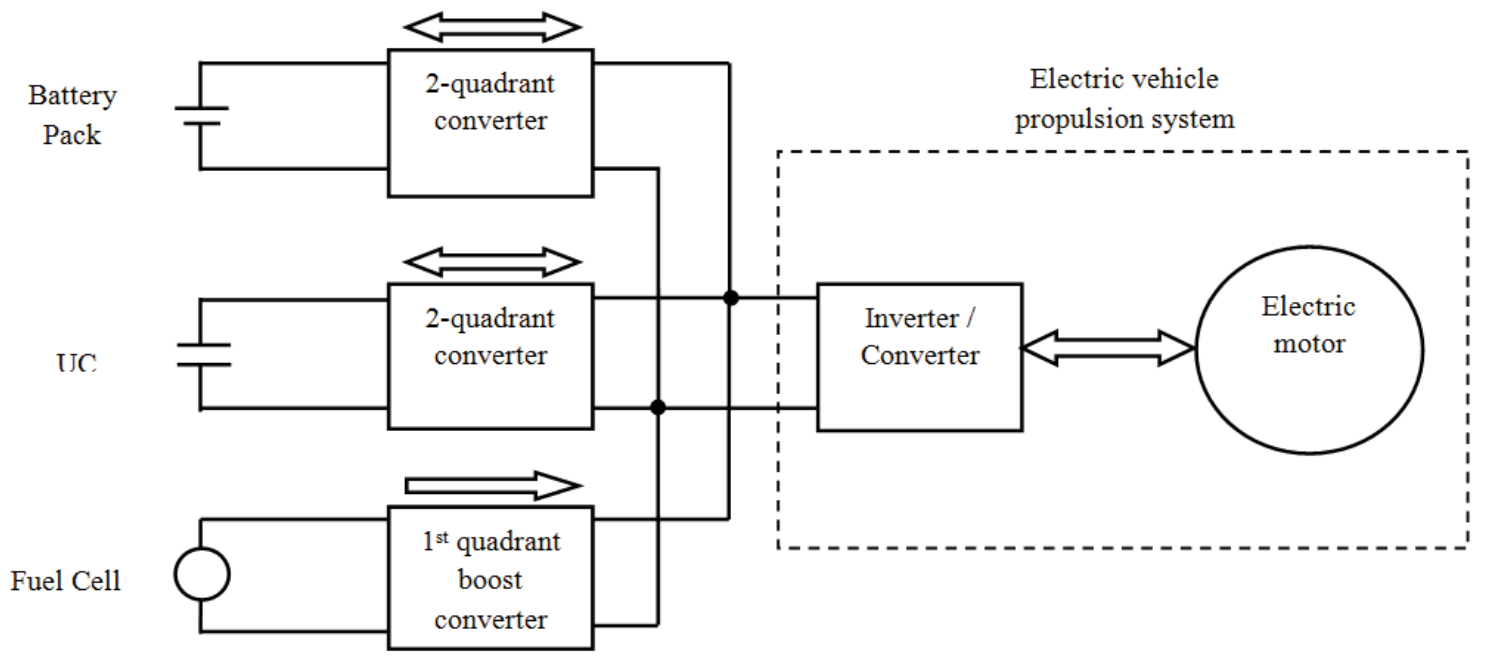

Fig. 2 Proposed parallel energy sharing system



Fig. 3 Proposed parallel energy-sharing control for the FC-battery-UC hybrid system

UC is used mainly for two reasons: to provide the peak power requirement during acceleration and to absorb vehicle kinetic energy during regenerative braking. It is therefore important to ensure that the UC is always ready to provide the peak power as well as to absorb the braking power. For this reason, the SOC of the $\mathrm{UC}$ is made dependent on the vehicle 
speed such that the available space of energy storage in the UC is proportional to the vehicle kinetic energy. For instance, if the vehicle is moving fast (i.e. large kinetic energy), more room is made available in the UC for regenerative braking and vice versa. Thus the UC voltage is given by equation (1).

$\mathrm{V}_{\mathrm{UC}}(\mathrm{U}) \leq \sqrt{\mathrm{V}_{\mathrm{UC}, \max }^{2}-\frac{\mathrm{M}}{\mathrm{C}_{\mathrm{UC}}} \mathrm{v}^{2}}$

In (1), $V_{U C}$ is the terminal voltage of the UC, $V_{U C, \max }$ is the allowable maximum voltage of the $\mathrm{UC}, M$ is the mass of the vehicle, $v$ is the speed of vehicle and $C_{U C}$ is the capacitance of the UC. To ensure that the UC always has an adequate energy from the battery and FC for vehicle acceleration, UC charging command $\left(I_{U C-C}\right)$ is added to the battery and FC current references. Conversely, UC need to be discharged to provide sufficient volume for the vehicle kinetic energy during regenerative braking. This can be realized by summing up the UC discharge command $\left(I_{U C-D}\right)$ to the UC current control loop. To avoid battery being charged by UC, the UC discharge command is limited to load current demand. In the proposed energy control system, battery is only charged by the FC and controlled through the battery charging control loop. A simple charging method is implemented to charge on the battery, which is based on constant current-constant voltage (CCCV) method. It is assumed that the initial SOC of the battery can be obtained based on its open-circuit terminal voltage [10]. The battery charging command $\left(I_{B a t-C}\right)$ is generated by adding output of the battery voltage controller loop with the current reference of FC current control loop.

Based on the discussion above, the reference signals for each control loop are summarized as below:

$$
\begin{aligned}
& V_{\text {bus ref }}=\text { constant } \\
& I_{U C \text { ref }}=I_{\text {load }}-\left(I_{F C \text { feedback }}+I_{\text {Batt feedback }}\right)+I_{U C-D} \\
& I_{\text {Batt ref }}=I_{\text {load }}+I_{U C-C}-I_{F C \text { feedback }} \\
& I_{F C \text { ref }}=I_{\text {load }}+I_{\text {Batt-C }}+I_{U C-C}
\end{aligned}
$$

Where $V_{\text {bus ref }}$ is the dc bus voltage, $I_{U C \text { ref }}, I_{\text {Batt ref }}$ and $I_{F C \text { ref }}$ are the current loop reference signals for the $\mathrm{UC}$, battery and $\mathrm{FC}$ respectively. $I_{\text {load }}$ is the load current demand, $I_{U C-C}$ and $I_{U C-D}$ is the UC charge and discharge signal that is generated from the UC voltage control loop, $I_{F C \text { feedback }}$ and $I_{\text {Batt feedback }}$ are the current feedback signals for the FC and battery respectively, and $I_{B a t-C}$ is the battery charging command.

\section{RESULTS AND DISCUSSIONS}

Simulations as well as experiments are carried out to verify the viability of the proposed method. The simulation is carried out using of MATLAB/Simulink simulation program. Fig. 4 shows the laboratory-scale experimental set-up used to verify the proposed scheme. In the experi ment, the battery module is composed of 4 series connected $12 \mathrm{~V}, 45 \mathrm{AH}$ calcium-calcium batteries. The UC used in the experiment is a BMOD0165 EO48 BO1 BOOSTCAP from Maxwell with 165F capacity and $48 \mathrm{~V}$ voltage rating. Due to the unavailability of the $\mathrm{FC}$ generator during the experiment, its behavior is emulated by a dc power supply HP6675A with an output voltage set to $48 \mathrm{~V}$. The DC bus voltage is regulated at $80 \mathrm{~V}$ and a closed-loop torque control DC motor drive rated at $0.25 \mathrm{hp} 120 \mathrm{~V} 3000$ r.p.m. is used to represent vehicle propulsion system. The control algorithm is implemented using dSPACE DS1104 controller board with an overall sampling period of $100 \mu \mathrm{s}$.

Fig. 5 and 6 show the simulation and experimental results during an ideal start-stop cycle, respectively. The DC motor is accelerated from stand still to a steady speed speed of 1300 rpm and then decelerated back to stand still. As can be seen from the figures, the simulation and experimental results are in close agreement. Initially, the battery is fully charged and the SOC of the UC is set at $87.5 \%\left(V_{U C}=42 \mathrm{~V}\right)$. During acceleration, the peak current is mainly supplied by the UC followed by the battery and FC. This can be clearly seen from Fig. 7(a), which is closer look of Fig. 6 during acceleration. It can also be seen that the slope of the current drawn from the battery and FC are limited; depending on the setting of time constants $\tau_{1}$ and $\tau_{2}$ which effectively limit the rate of change of the reference current. During steady state speed, UC is discharged to make room for the braking power. Once the UC attained to its reference voltage, depending on the required power under steady state speed, FC supplies all the constant load power while the battery and UC operate in idle conditions. From Fig. 7 (b), when the DC motor decelerates, it can be observed that a sharp braking power was mostly recuperated by the UC followed by the battery (within a limited current slope). Subsequently, the battery and FC will charge the UC up to its reference voltage to ensure good dynamic response later during acceleration.

\section{CONCLUSION}

This paper mainly discusses on design and control structures for the proposed parallel energy-sharing containing FC generator, battery and UC. Through the proposed energy control algorithm, overstress toward FC and battery is avoided. Voltage of the UC is controlled accordingly to vehicle speed in order to ensure sufficient energy for vehicle acceleration and also adequate volume for vehicle braking. The proposed method does not guarantee perfect results in all situations, but provides a satisfactory energy management method in controling the overall FCHV system. The validity of the proposed energy control scheme is supported by simulation and experimental results under average and peak power response. 


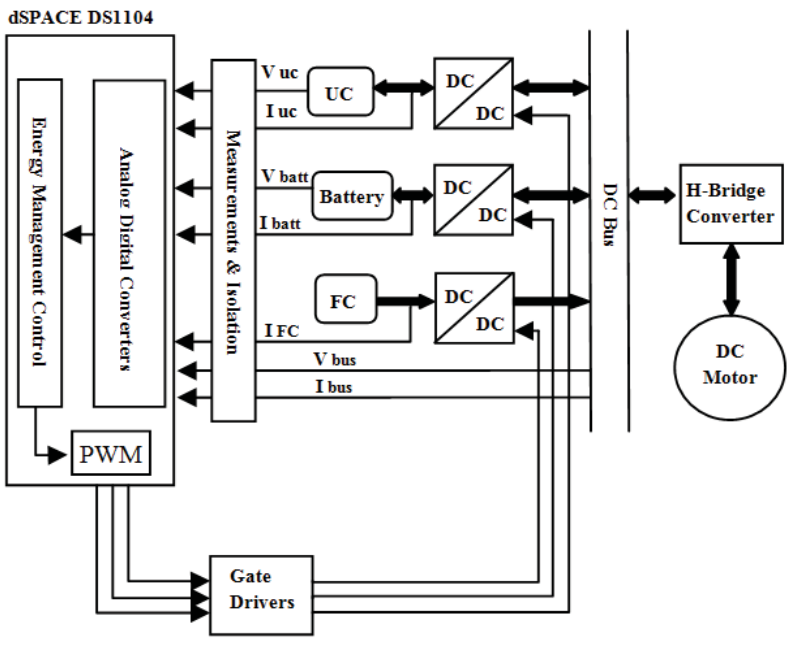

a)

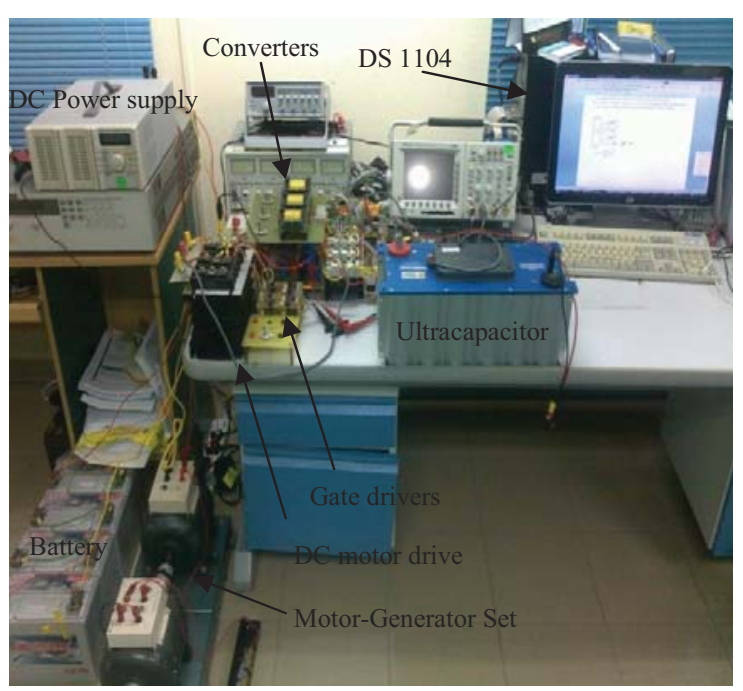

b)

Fig. 4 Proposed implementation (a) Block diagram of the experimental set-up (b) Picture of complete experimental set-up
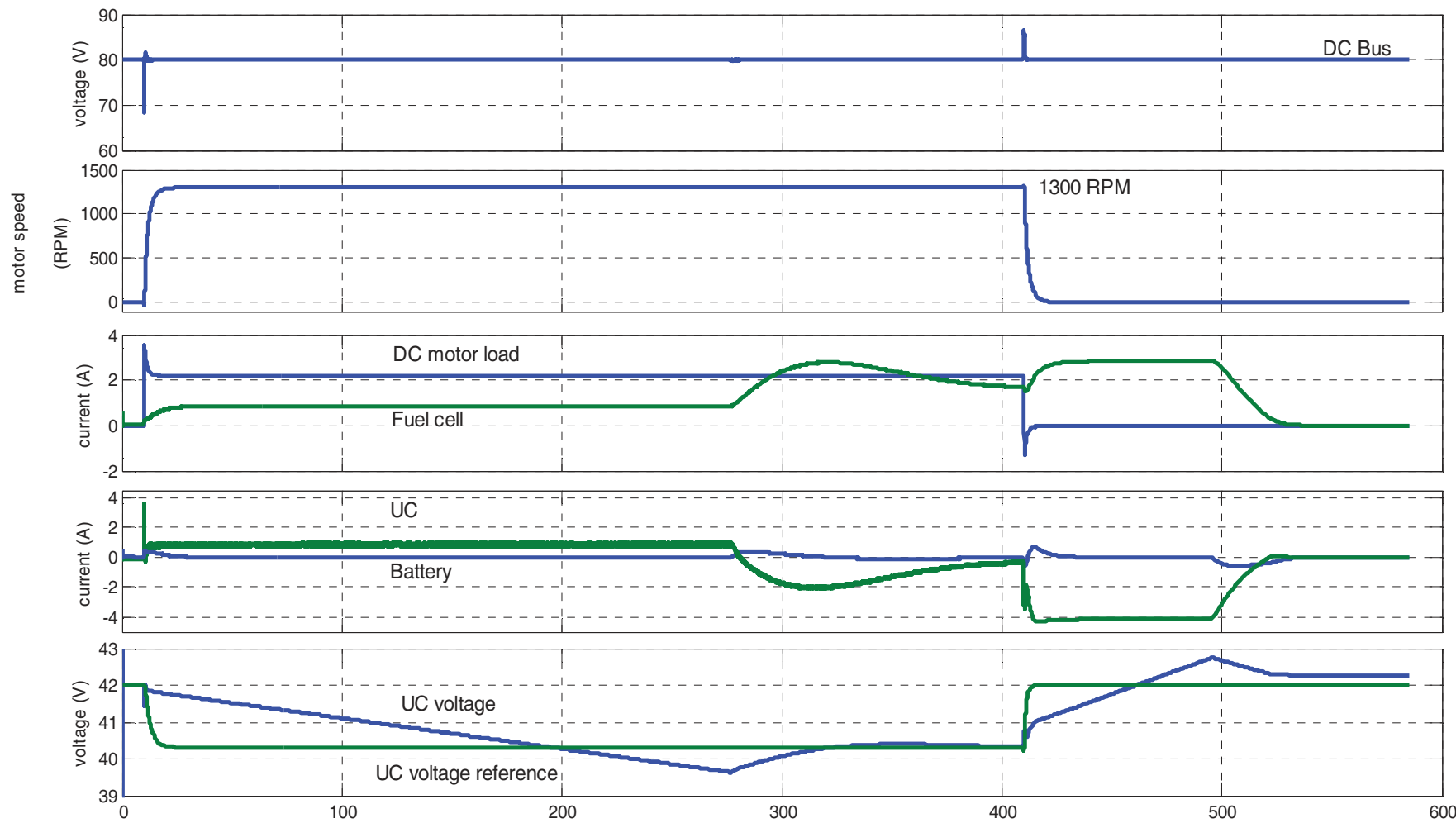

Fig. 5 Simulation results for an ideal start-stop cycle 


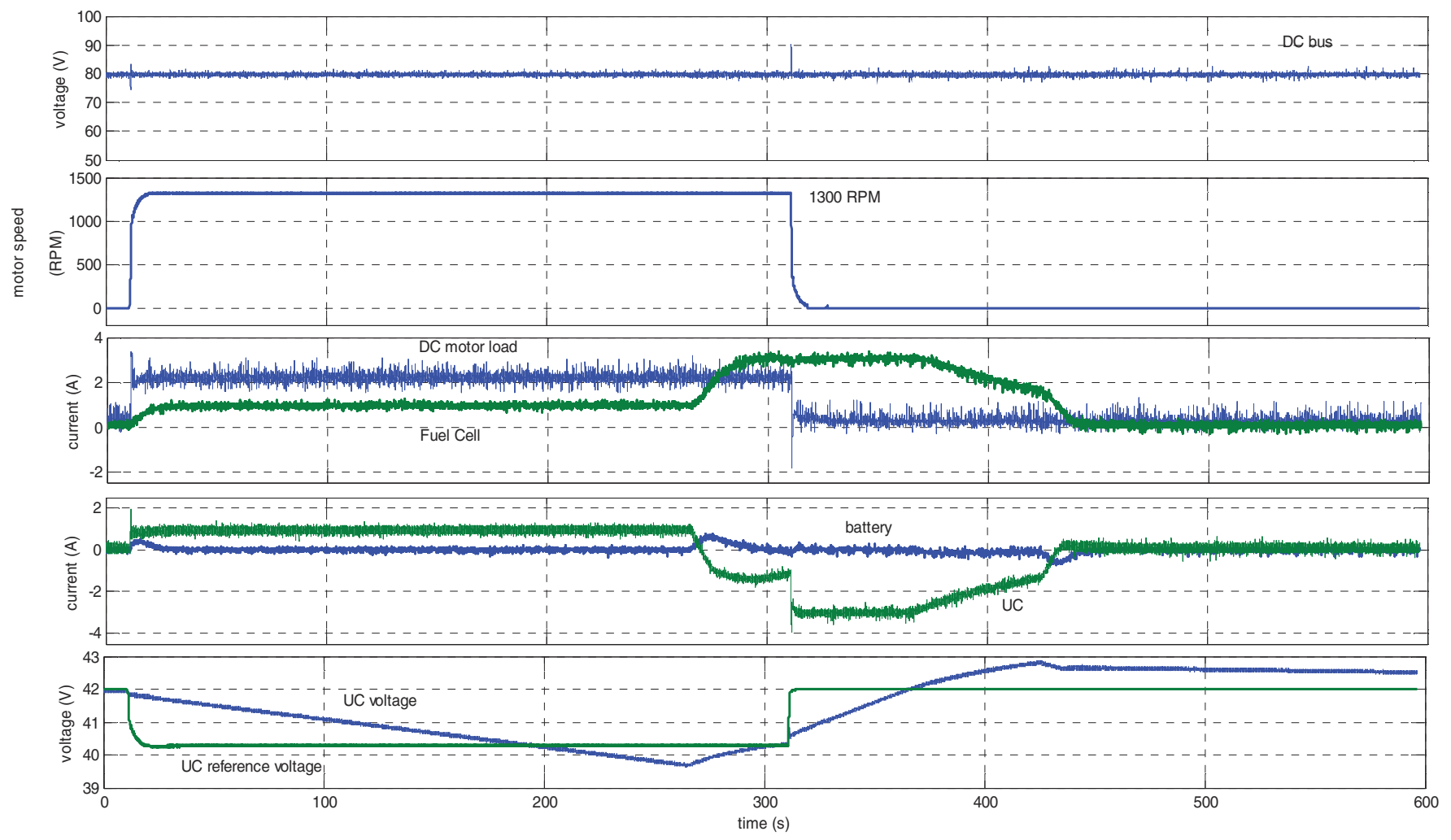

Fig. 6 Experimental results for an ideal start-stop cycle

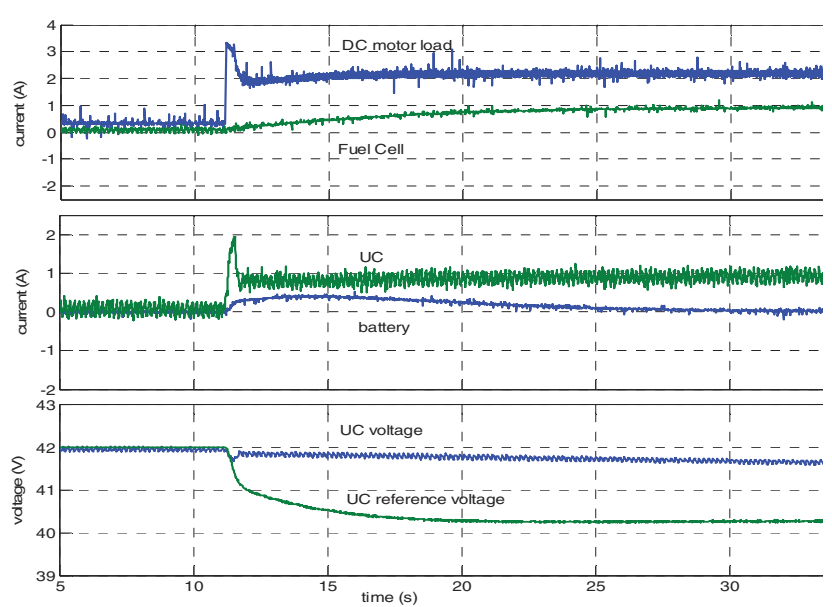

a)

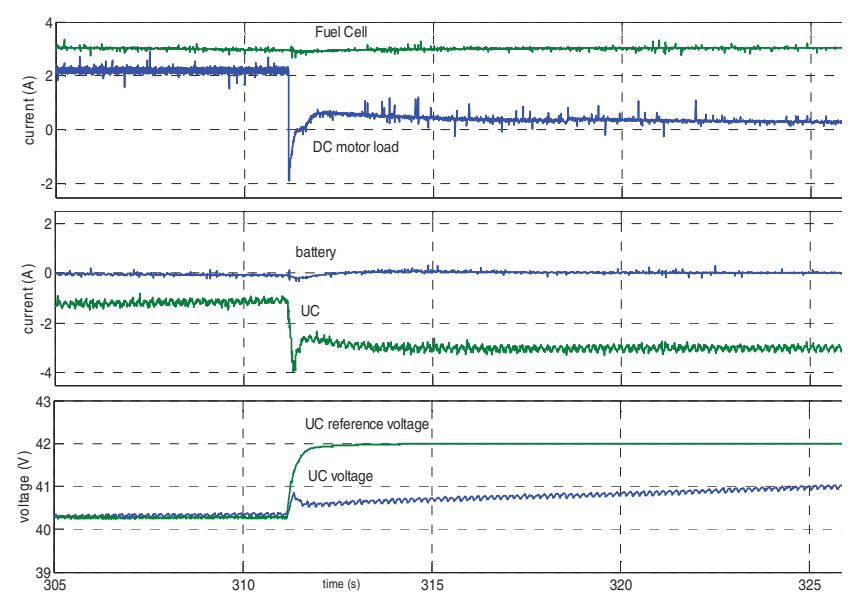

b)

Fig. 7 a) Transient responses during motor acceleration b) Transient responses during motor deceleration (zoom in)

\section{ACKNOWLEDGEMENT}

The authors would like to thank the Ministry of Higher Education of Malaysia and Universiti Teknologi Malaysia for funding this research

\section{REFERENCES}

[1].Phatiphat Thounthong, StephaneRael, Bernard Davat (2009), "Energy management of fuel cell/ battery/ supercapacitor hybrid power source for vehicle applications", Journal of Power Sources, Vol. 193, Issue 1, pp. 376-385, Aug 2009.

[2].WenzhongGao (2005), "Performance Comparison of a Fuel Cell-Battery Hybrid Powertrain and a Fuel Cell- 
Ultracapacitor Hybrid Powertrain", IEEE Transactions on Vehicular Technology, Vol. 54, No.3, pp. 143-150, May 2005.

[3].Jennifer Bauman and Mehrdad Kazerani (2008), "A Comparative Study of Fuel Cell-Battery, Fuel cellUltracapacitor, and Fuel Cell-Battery-Ultracapacitor Vehicles", IEEE Transactions On Vehicular Technology, Vol. 57, No. 2, pp. 760, March 2008.

[4].N. Schofield, H. T. Yap and C. M. Bingham (2005), "Hybrid Energy Sources for Electric and Fuel cell Vehicle Propulsion", in IEEE 2005 Vehicle Power and Propulsion Conference, 2005, pp. 522-529.

[5].E. Schaltz, A. Khaligh and P. O. Rasmussen, "Investigation of Battery/ Ultracapacitor Energy Storage Rating for a Fuel Cell Hybrid Electric Vehicle", in IEEE 2008 Vehicle Power and Propulsion Conference, 2008, pp.1-6.

[6].Phatiphat Thounthong, Stephane Real and Bernard Davat, "Control Strategy of Fuel cell/ Supercapacitors Hybrid
Power Sources for Electric Vehicle", Journal of Power Sources, Vol. 158, Issue 1, pp. 806-814, July 2006.

[7].C. M. Jefferson and R. H. Barnard (2002). Hybrid Vehicle Propulsion. Southampton, UK: WIT Pr.

[8].M.Ehsani, Y.Gao, and A. Emadi (2010). Modern Electric, Hybrid Electric and Fuel Cell Vehicle-Fundamentals, Theory and Design (Second Edition). Boca Raton: CRC press.

[9]. Society of Automotive Engineers (2002). Advanced Hybrid Vehicle Powertrain Technology. Warrendale, PA: Society of Automotive Engineer.

[10]. A. A. Ferreira, J. A. Pomilio, G. Spiazzi and L. de Araujo Silva, "Energy Management Fuzzy Logic Supervisory for Electric Vehicle Power Supplies System", IEEE Transactions on Power Electronics, Vol. 23, No. 1, pp. 107-115, Jan 2008. 